WORKSHOP ON EDUCATIONAL INNOVATION

IN ARCHITECTURE JIDA'17

JORNADES SOBRE INNOVACIÓ

DOCENT EN ARQUITECTURA JIDA'17

ESCUELA TÉCNICA SUPERIOR DE ARQUITECTURA DE SEVILLA 16 Y 17 DE NOVIEMBRE DE 2017 


\section{GILDA}

Organiza e impulsa GILDA (Grupo para la Innovación y Logística Docente en la Arquitectura), en el marco del proyecto RIMA (Investigación e Innovación en Metodologías de Aprendizaje), de la Universitat Politècnica de Catalunya - BarcelonaTech (UPC) y el Institut de Ciències de l'Educació (ICE). https://www.upc.edu/rima/ca/grups/gilda

\section{Editores}

Daniel García-Escudero, Berta Bardí i Milà

\section{Revisión de textos}

Rodrigo Carbajal Ballell, Silvana Rodrigues de Oliveira, Jordi Franquesa

\section{Edita}

Iniciativa Digital Politècnica Oficina de Publicacions Acadèmiques Digitals de la UPC

ISBN 978-84-9880-681-6 (UPC)

elSSN 2462-571X

D.L. B 9090-2014

(C) de los textos y las imágenes: los autores

(C) de la presente edición: Iniciativa Digital Politècnica Oficina de Publicacions Acadèmiques Digitals de la UPC 


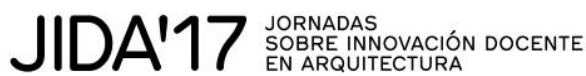

\section{Comité Organizador JIDA'17}

Dirección, coordinación y edición

\section{Berta Bardí i Milà (GILDA)}

Dra. Arquitecta, Departamento de Proyectos Arquitectónicos, ETSAV-UPC

Daniel García-Escudero (GILDA)

Dr. Arquitecto, Departamento de Proyectos Arquitectónicos, ETSAB-UPC

\section{Organización}

Rodrigo Carbajal Ballell (humAP)

Dr. Arquitecto, Departamento de Proyectos Arquitectónicos, ETSA-US

Jordi Franquesa (Coordinador GILDA)

Dr. Arquitecto, Departamento de Urbanismo y Ordenación del Territorio, ETSAB-UPC

Joan Moreno Sanz (GILDA)

Dr. Arquitecto, Departamento de Urbanismo y Ordenación del Territorio, ETSAV-UPC

Silvana Rodrigues de Oliveira (humAP)

Arquitecta, Departamento de Proyectos Arquitectónicos, ETSA-US

Judit Taberna (GILDA)

Arquitecta, Departamento de Representación Arquitectónica, ETSAB-UPC

\section{Comité Científico JIDA'17}

\section{Rodrigo Almonacid Canseco}

Dr. Arq., Dpt. de Teoría de la Arquitectura y Proyectos Arquitectónicos, ETSA Valladolid

Fernando Álvarez Prozorovich

Departamento de Historia y Comunicación, ETSAB-UPC

\section{Atxu Amann Alcocer}

Dra. Arquitecta, Departamento de Proyectos Arquitectónicos, ETSAM-UPM

\section{Silvia Blanco}

Dra. Arquitecta, Centro Superior de Estudios de Galicia, Universidad San Jorge 


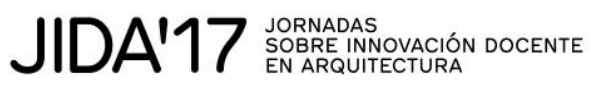

\section{Ivan Cabrera i Fausto}

Dr. Arq., Dpt. de Mecánica de los Medios Continuos y Teoría de Estructuras, ETSA-UPV

\section{Raúl Castellanos Gómez}

Dr. Arquitecto, Departamento de Proyectos Arquitectónicos, ETSA-UPV

\section{Nuria Castilla Cabanes}

Dra. Arquitecta, Departamento de Construcciones arquitectónicas, ETSA-UPV

\section{Eduardo Delgado Orusco}

Dr. Arquitecto, Departamento de Proyectos Arquitectónicos de la Universidad de Zaragoza

\section{Mariona Genís Vinyals}

Dra. Arquitecta, BAU Centro Universitario del Diseño de Barcelona

\section{María González}

Arquitecta, Departamento de Proyectos Arquitectónicos, ETSA-US

\section{Antonio Juárez Chicote}

Dr. Arquitecto, Departamento de Proyectos Arquitectónicos, ETSAM-UPM

\section{Juanjo López de la Cruz}

Arquitecto, Departamento de Proyectos Arquitectónicos, ETSA-US

\section{Nieves Mestre}

Dra. Arquitecta, Departamento de Proyectos Arquitectónicos, Universidad Europea

\section{Francisco Javier Montero}

Dr. Arquitecto, Departamento de Proyectos Arquitectónicos, ETSA-US

\section{Antonio Peña Cerdán}

Arquitecto, Departamento de Proyectos Arquitectónicos, ETSA-UPV

\section{Ana Portalés Mañanós}

Dra. Arquitecta, Departamento de Urbanismo, ETSA-UPV

\section{Amadeo Ramos Carranza}

Dr. Arquitecto, Departamento de Proyectos Arquitectónicos, ETSA-US

\section{Jaume Roset Calzada}

Dr. Físico, Departamento de Física Aplicada, ETSAB-UPC

José Vela Castillo

Dr. Arquitecto, IE School of Architecture and Design, IE University (Segovia, Spain) 


\title{
Del cuadrado al cubo: metodología para la comprensión de la Arquitectura
}

\section{From the square to the cube: a methodology for understanding Architecture}

\author{
Díaz-Segura, Alfonso; Ros-Campos, Andrés; Ábalos-Ramos, Ana; Juan-Ferruses, Ignacio \\ Departamento Proyectos, Teoría y Técnica del Diseño y la Arquitectura, \\ Universidad Cardenal Herrera-CEU, CEU Universities, España, \\ alfonsodiaz@uchceu.es; roscampos@uchceu.es; ana.abalos@uchceu.es; ignacio.juan@uchceu.es
}

\begin{abstract}
Architecture is an integrative activity in which art and technique, matter and emptiness, past and future, technology and crafts, individual and society coexist. At times the teaching of the first year has been divided into subjects that appear to the student as disconnected islands. The aim of the present text is to propose transversal exercises that allow building bridges between them that facilitate the student the global understanding of Architecture.
\end{abstract}

Keywords: Architecture, methodology, learning, integration, innovation.

\begin{abstract}
Resumen
La Arquitectura es una actividad integradora en la que conviven arte y técnica, materia y vacío, pasado y futuro, tecnología y artesanía, individuo y sociedad. En ocasiones la enseñanza del primer curso se ha troceado y distribuido en asignaturas que aparecen ante el alumno como islas inconexas. La voluntad del presente texto es proponer unos ejercicios transversales que permitan tender puentes entre ellas que faciliten al alumno la comprensión global de la Arquitectura.
\end{abstract}

Palabras clave: Arquitectura, metodología, enseñanza, integral, innovación.

Bloque temático: Metodologías activas (MA) 


\section{Objetivos}

El objetivo de este artículo es proponer una metodología que permita aproximar las asignaturas del primer curso de la carrera a la arquitectura, a través de ejercicios que las pongan en relación con ejemplos y temáticas puramente arquitectónicas, y también entre ellas mismas.

Del cuadrado al cubo es una metáfora sobre la enseñanza de la arquitectura. Pasar de la figura bidimensional al objeto tridimensional implica varias consecuencias: incorporar una tercera dimensión en la representación gráfica, comprender la verdadera naturaleza del espacio, establecer relaciones bidireccionales entre geometría plana y volumen, o entender que puede haber distintas aproximaciones al mismo objeto.

Esta última experiencia debería darse en cada una de las asignaturas que integran los estudios oficiales de Arquitectura: acercarse a ella desde distintas disciplinas y áreas de conocimiento, si bien se detecta cierta dificultad en algunas de ellas, y en especial en las del primer curso.

\section{Introducción}

Aprovechando la transformación que en los últimos años ha experimentado nuestra Escuela de Arquitectura, integrada en la Escuela Superior de enseñanzas Técnicas de la Universidad Cardenal Herrera de Valencia, era una buena oportunidad de coordinar el esfuerzo pedagógico del profesorado con el objetivo de ajustar el contenido de la docencia a una realidad multicultural compleja y a la necesidad, percibida históricamente de vincular con mayor fuerza las asignaturas de primer curso entre sí y con la disciplina en general.

Esta doble Interacción, entre asignaturas del mismo curso y cursos superiores, otorga comprensión del recorrido a realizar y ayuda a enfocar las asignaturas con el interés necesario para superarlas. Por tanto otro objetivo es la Integración de metodologías y coordinación entre distintos profesores de distintas áreas de conocimiento detectando necesidades docentes y orientando el esfuerzo pedagógico para hacer comprender al alumno de forma integral la disciplina de la arquitectura.

Desde el punto de vista arquitectónico, se pretende que la coherencia de contenidos y la posibilidad de asociación de los mismos entre materias, potencie las habilidades adquiridas por el alumnado. Para ello el profesorado propone diseñar una serie de tareas que faciliten la comprensión, por parte del alumno, de la complejidad espacial y transmitan la certeza de la dificultad del proceso creativo en la arquitectura.

En definitiva, se postula una reflexión de ejercicios propuestos para que el mensaje docente sea mucho más potente. Esta tarea, lejos de lo que pudiera parecer, se presenta como algo profundo, ya que el profesorado debe diseñar correctamente estas tareas con el fin de sintetizar los contenidos y ayudar a su comprensión de la forma más eficaz posible. Requiere por tanto la agudeza adquirida por la experiencia y la originalidad suficientes como para incentivar a su vez el espíritu creativo. Esta cuestión justifica el recurrir a experiencias docentes históricas vinculadas a la arquitectura con el fin de promover la reflexión del alumno: nuestro reto es invitarles y facilitarles el pensamiento.

Nos preocupa también que nuestros alumnos alcancen un nivel de dominio del lenguaje gráfico profesional, con el fin de dominar la representación tanto en dos como en tres dimensiones. Alcanzar la visión tridimensional se descubre como fundamental para generar espacio arquitectónico. En este sentido se diseñan algunas experiencias docentes que integran este objetivo. 
En las conversaciones mantenidas entre el profesorado de las distintas áreas de conocimiento, se ha perseguido detectar y valorar la estructura básica de la forma desde los puntos de vista de las distintas materias.

Así se hace necesaria una acertada elección de los ejercicios a realizar, que integren los diferentes conocimientos tratados por el estudiante de arquitectura en su primer año de formación académica, y despierte su interés por la aplicación de materias básicas, como Matemáticas, Física o Dibujo, en el proceso creativo, y refuercen la relación entre asignaturas que a menudo permanecen como islas en el plan de estudios.

\section{El Grado en Fundamentos de Arquitectura}

El diseño del plan de estudios de Arquitectura -enmarcado en la voluntad de definir un itinerario de 5+1 años integrando el Grado y el Máster habilitante-, supuso en la Universidad Cardenal Herrera-CEU (en adelante UCH-CEU) una oportunidad para cambiar la concepción tradicional de asignaturas como cajas estancas. Además, la concepción genérica del EEES conocido comúnmente como "Bolonia", implicaba repensar la forma en que veníamos enseñando arquitectura en las Escuelas españolas, no tanto en los resultados obtenidos por los alumnos, sino en la metodología empleada. No cabe duda de que la enseñanza y el ejercicio de la Arquitectura española goza del reconocimiento internacional (posiblemente más que el nacional) desde hace años. Por consiguiente, podríamos decir que la formación era y sigue siendo buena. El cambio venía más bien provocado por la introducción de una serie de conceptos pedagógicos que parecían dificultar la dinámica habitual de las clases; muchos de esos conceptos ya se aplicaban de forma intuitiva en las asignaturas, especialmente las proyectuales, pero había otras en las que probablemente la resistencia al cambio sería mayor. De modo que el reto consistía en la organización de un plan de estudios claramente estructurado en base a una disección del proceso formativo en competencias, actividades formativas, y resultados de aprendizaje. El protagonista ya no era el profesor demostrando su saber, sino el alumno certificando su adquisición de conocimiento.

Sin embargo, si bien la propia estructura del sistema ayudaba a organizar la secuencia de asignaturas y sus competencias, corre el riesgo de asignar dichas competencias y los resultados de aprendizaje de forma autónoma para cada una de ellas. Un mal entendimiento del fin último de la enseñanza, podría conducir a considerar que las asignaturas de construcción, el alumno no adquiriese ninguna competencia gráfica, o que en las asignaturas de proyectos no se esperasen resultados relacionados con estructuras, por ejemplo. Es decir, que el mismo marco pedagógico general propuesto era una herramienta muy útil para clarificar resultados esperables, pero al mismo tiempo entrañaba un riesgo al configurar las asignaturas como islas.

De ahí la importancia de considerar los nuevos planes de estudios como una oportunidad, y las disposiciones legales que regulan los estudios conducentes al título de arquitecto como una inestimable ayuda, pero considerando cuáles eran sus límites.

\section{El primer curso de Arquitectura}

Si esto podía detectarse en cualquier curso del Grado, la situación es más delicada si cabe en el primero de ellos, dado que debemos incorporar unas materias básicas propias de la rama de conocimiento a la que se adscriben nuestros estudios, que en España es la de Ingeniería y Arquitectura. De hecho gran parte de estas asignaturas ni siquiera serían tenidas en cuenta en foros sobre enseñanza de arquitectura, ya que se trata de asignaturas "complementarias" o 
"genéricas", "transversales"...en definitiva "al margen" de la formación estrictamente específica de un arquitecto.

El motivo es que la dispersión de temáticas es muy grande y la conexión con la arquitectura suele ser débil (Matemáticas, Física, o Geometría Descriptiva). No nos referimos a que esas materias no alimenten la arquitectura, eso sería un grave error conceptual; nos referimos a que dicha relación no aparecía hasta uno o dos años después y de forma tangencial.

Además se trata de asignaturas que tradicionalmente se han desenvuelto de un modo determinado y hay una inercia que dificulta su transformación. Se ha recorrido ya un camino en la introducción de nuevas metodologías como el aprendizaje basado en proyectos, o el método del caso, que parecen casi obvios para la enseñanza de la arquitectura. La novedad, por tanto, no reside ahí, sino en el diseño y ejecución de una estrategia conducente a disolver los límites entre asignaturas del primer curso.

\section{Planificación de las acciones}

Para organizar dicha estrategia se han buscado los puntos de encuentro de asignaturas y profesores, según la siguiente hoja de ruta:

1. Designación de asignaturas implicadas.

Serán todas las del $1^{\circ}$ curso del Grado en Fundamentos de Arquitectura de la $\mathrm{UCH}-$ CEU: Matemáticas I, Física I, Geometría Descriptiva I, Dibujo Arquitectónico II, Historia del Arte, Matemáticas II, Física II, Geometría Descriptiva II, Dibujo Arquitectónico II, e Introducción a la Arquitectura.

2. Puntos de contacto de cada asignatura con la Arquitectura.

La segunda fase se basa en establecer qué puentes se pueden tender entre las asignaturas del primer curso, y la arquitectura. A modo de resumen clasificatorio pero no limitativo, proponemos:

- Matemáticas I: Orden, series, proporciones, precisión.

- Física I: Estabilidad, comportamiento mecánico.

- Geometría Descriptiva I: Figuras, representación bidimensional y tridimensional.

- Dibujo Arquitectónico I: Expresión gráfica, proporciones, escala.

- Historia del Arte: Memoria, forma, expresión, lenguaje, espacio.

- Matemáticas II: Cálculo, herramientas para física y estructuras, formulación de geometría.

- Física II: Acústica, térmica, herramientas para instalaciones,

- Geometría Descriptiva II: Expresión gráfica, superficies complejas.

- Dibujo Arquitectónico II: Códigos, representación, grafismo, proporción, métrica, escala.

- Introducción a la Arquitectura: Lugar, forma, espacio, materia, color, luz, programa, memoria. 
3. Reuniones de comunicación y puesta en común de objetivos y metodologías entre los profesores.

Una vez detectados los puntos de encuentro con asignaturas del mismo semestre, el reto es definir ejercicios comunes para las dos asignaturas, que precisen de la concurrencia de los profesores y del manejo de conocimientos adquiridos en todas ellas. Para abordar racionalmente la tarea, se va a acotar a aquellas asignaturas entre las que los vínculos son más potentes. De este modo se clarificará el objetivo, se limitará el número de competencias a adquirir, se controlará la complejidad del ejercicio, se podrá hacer más tangible el resultado, y se dejará la puerta abierta a futuras incorporaciones de otras asignaturas. Por consiguiente, la conclusión fundamental de la tercera fase fue que se generaría un catálogo de ejercicios vinculando unas asignaturas con otras, pero no necesariamente todas entre sí. Además, se decidió que sería interesante trabajar simultáneamente en dos dimensiones: la de las asignaturas concurrentes en el mismo semestre, y la de aquellas que se producían en semestres sucesivos.

Cuando se tratara de asignaturas del mismo semestre, cada asignatura propondría un ejercicio transversal que afectara a otras, y que dependiendo de la asignatura que liderase la acción, sería distinto. Es decir, si Matemáticas I propone un ejercicio que afecta a Geometría Descriptiva I e Historia del Arte, aquél otro propuesto por Geometría Descriptiva o Historia del Arte no debe necesariamente afectar a las mismas asignaturas, ni tener el mismo enunciado.

Cuando se tratase de asignaturas de distinto semestre, el enfoque sería un poco distinto, para contemplar la imposibilidad de coexistir en el tiempo; no se trataría tanto de compartir un ejercicio, como de hacer referencia a contenidos del temario, competencias adquiridas por el alumno, o resultados obtenidos de ejercicios previos o futuros. Mirar hacia delante o hacia atrás, según el semestre en que estuviéramos, para seguir trenzando las asignaturas entre ellas.

4. Puntos de contacto entre asignaturas del mismo y de distinto semestre.

El detalle de los cruces de contenidos y ejercicios propuestos puede verse en la tabla resumen adjunta.

5. Puesta en común de propuestas y revisión final.

Una vez propuestos los ejercicios por parte de cada unidad docente, se realizó una reunión para ponerlos en común y ajustarlos a las necesidades y las posibilidades de cada asignatura y profesor. También emanó el compromiso de redactar un enunciado completo para cada ejercicio, señalando objetivos, recursos, calendario y resultados esperados.

De los puntos de contacto tejidos entre las asignaturas, se derivó una red de relaciones y ejercicios que se organiza para su mejor entendimiento en forma de matriz, en la página siguiente. Asimismo, había que ajustar la estrategia a la logística disponible en la Escuela, por lo que se decidió ir implantando la matriz de forma progresiva curso a curso, según el siguiente calendario: 


\section{Curso 2016-17}

Planteamiento de la estrategia y redacción del plan de acción.

\section{Curso 2017-18}

Implantación de los siguientes ejercicios en el mismo semestre:

- Matemáticas I $\left(1^{\circ} \mathrm{s}\right)$

- Dibujo Arquitectónico I $\left(1^{\circ} \mathrm{s}\right)$

- Geometría Descriptiva II $\left(2^{\circ} \mathrm{s}\right)$

- Introducción a la Arquitectura $\left(2^{\circ} \mathrm{s}\right)$

Implantación de los siguientes ejercicios en distintos semestres:

- Matemáticas I $\left(1^{\circ} \mathrm{s}\right)$

- Dibujo I (10 $\mathrm{s})$

- Geometría Descriptiva II $\left(2^{\circ} \mathrm{s}\right)$

- Introducción a la Arquitectura $\left(2^{\circ} \mathrm{s}\right)$

\section{Curso 2018-19}

Implantación de los siguientes ejercicios en el mismo semestre:

- Física I $\left(1^{\circ} \mathrm{s}\right)$

- Geometría Descriptiva I $\left(1^{\circ} \mathrm{s}\right)$

- Historia del Arte $\left(1^{\circ} \mathrm{s}\right)$

- Dibujo Arquitectónico II ( $\left.2^{\circ} \mathrm{s}\right)$

- Matemáticas II $\left(2^{\circ} \mathrm{s}\right)$

- Física II $\left(2^{\circ} \mathrm{s}\right)$

Implantación de los siguientes ejercicios en distintos semestres:

- Física I $\left(1^{\circ} \mathrm{s}\right)$

- Geometría Descriptiva I $\left(1^{\circ} \mathrm{s}\right)$

- Historia del Arte $\left(1^{\circ} \mathrm{s}\right)$

- Dibujo Arquitectónico II $\left(2^{\circ} \mathrm{s}\right)$

- Matemáticas II $\left(2^{\circ} \mathrm{s}\right)$

- Física II $\left(2^{\circ} \mathrm{s}\right)$

\section{Metodología y matriz general de las acciones}

A la necesidad del estudiante novel por descubrir las claves del diseño arquitectónico, se le une la inquietud docente del profesorado de primer curso por proponer que aporte una mayor comprensión de los estudios de Arquitectura como una actividad inclusiva formada por áreas de conocimiento muy diversas. La metodología propone implicar al menos a tres asignaturas por semestre y lograr que desde todas ellas exista un vínculo para alimentar el proceso creativo de la arquitectura. Todo ello exige la preparación específica de contenidos en cada materia para proponer objetivos comunes y ejercicios que guíen al alumno entrelazando medios y fines académicos. No se trata tanto de saber, en el sentido de estar instruido en algo, como de aprender, en el sentido de participar activamente de la adquisición del conocimiento. Por tanto, el alumno construye su conocimiento a través de la acción.

A continuación se aporta una matriz en la que pueden observarse los puntos de contacto entre asignaturas y los ejercicios a que dan lugar. 


\section{Matriz general}

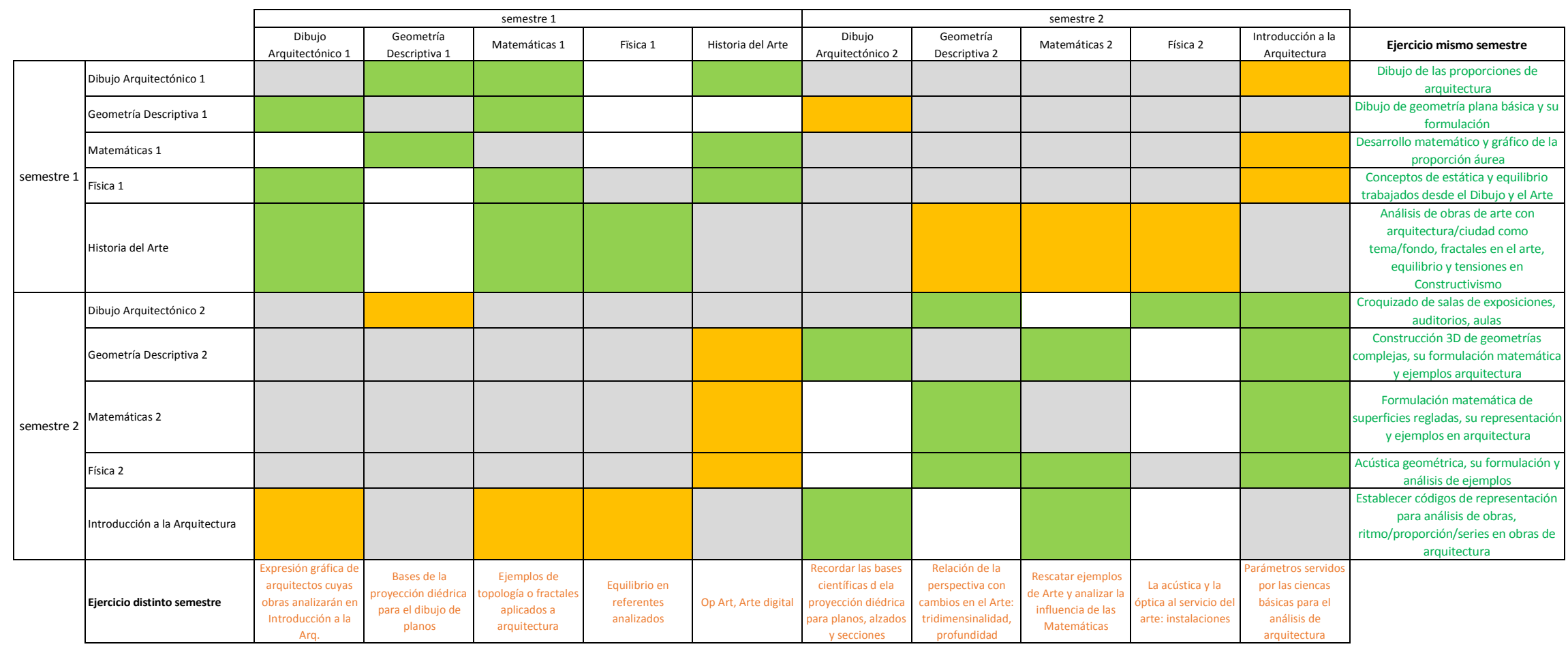

Fig 1. En esta figura se resume las relaciones establecidas entre asignaturas del mismo y de distinto semestre, así como los ejercicios a que darían lugar. Entrando en horizontal se entrelazan asignaturas del mismo semestre, señalando en verde los puntos de contacto. Entrando en vertical se señala en color naranja los contactos entre asignaturas de distinto semestre. En color blanco se dejan posibles cruces a investigar en el futuro. En color gris, cruces desestimados o imposibles. 


\section{Caso práctico}

La metáfora del paso del cuadrado al cubo alude a la evolución del alumno en su comprensión del espacio, evidenciando los aportes de cada asignatura que nos permiten pasar de lo bidimensional a lo tridimensional, del encuadre del dibujo, al encaje de la maqueta. Mediante la implicación de asignaturas como matemáticas, física, dibujo, geometría descriptiva o historia del arte. En la trayectoria de estos últimos años se ha puesto en práctica este tipo de metodología con resultados satisfactorios. Creemos que los resultados obtenidos invitan a seguir trabajando sobre este tipo de enfoques.

Por destacar un caso concreto proponemos centrarnos en el problema del hecho complejo de la morfología arquitectónica. Durante el siglo pasado las teorías en torno a la geometría fractal ${ }^{1}$ enunciaban que la aparente aleatoriedad se basaba en procesos reiterativos, geometrizables y repetitivos. Extrapolando esta idea a la arquitectura, cabría pensar en aquellas arquitecturas aparentemente complejas cuyo análisis descubría que se fundamentaban en el uso de una estructura básica y elemental.

En definitiva, un campo a explorar y en torno al cual generar las primeras propuestas docentes en la línea que nos interesaba, que era la estructura formal de la arquitectura. En esta reflexión nos invade el recuerdo de las experiencias de los años 60 en la Cooper Union ${ }^{2}$ de Nueva York, cuyo ejemplo pedagógico invita a pensar sobre la manera óptima de hacer entender al alumno el proceso creativo. Más recientemente, diseños arquitectónico como "la casa final de madera" (Final Wooden House) $^{3}$ diseñada por Sou Fujimoto en Japón nos demuestran que partiendo de una sencillez extrema es posible alcanzar morfologías complejas.

\section{Ejemplo desarrollado en aula}

En el ejemplo de ensayo que se explica a continuación y desarrollado durante el curso 20162017, se involucran las asignaturas de Historia del Arte, Dibujo Arquitectónico, Matemáticas y Geometría Descriptiva. El enfoque del ejercicio comienza con la visita de la arquitectura de Antoni Gaudí, que como excusa ofrece un ejemplo emblemático del uso de superficies regladas. Esta relación con la realidad diseñada y construida sirve de introducción para un análisis de su complejidad descodificada a través de la geometría dibujada, el conocimiento de su expresión matemática o su comportamiento físico.

Consecuentemente la primera toma de contacto se realiza desde el punto de vista histórico y de la contextualización de la obra de Gaudí en la historia de la arquitectura, posteriormente se analiza el comportamiento físico y estructural de sus soluciones a través de ejercicios prácticos consistentes en la construcción de modelos físicos que permiten estudiar la estructura. Seguidamente, desde el área de Matemáticas, se estudia la formulación de ecuaciones y su alteración al variar cada uno de sus parámetros, para posteriormente desde Geometría Descriptiva construir las superficies regladas de forma que se aúnen conocimientos y se convierta lo que podría ser un aprendizaje abstracto en algo tangible que favorece la comprensión del problema y su solución.

\footnotetext{
1 Benoît Mandelbrot. 1924-2010. Matemático que estudió las geometrías fractales explicadas en su libro Fractal Geometry of Nature, en 1982.

2 The Cooper Union for the Advancement of Science and Art, es una universidad privada ubicada en Nueva York y se considera como una de las más prestigiosas de Estados Unidos.

3 Final Wooden House. Sou Fujimoto. 2005-2008. Kumamoto, Japón
} 


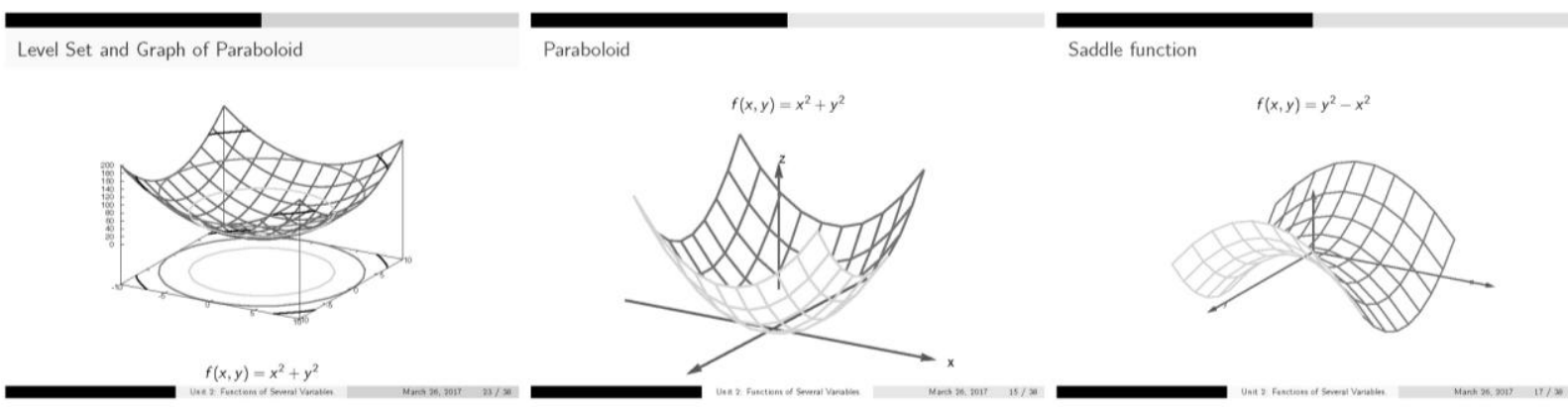

Fig 2. Formulación matemática y representación del paraboloide y del paraboloide hiperbólico. Dra Lucía Hilario. Universidad Cardenal Herrera CEU.

La práctica propone considerar las superficies regladas inscritas en un cubo. De este modo se visualiza gráficamente las posibilidades de variación del resultado al alterar alguno de los parámetros como es la posición de las nudos y por tanto la longitud de las barras. Una vez dibujados los paraboloides hiperbólicos, hiperboloides, conoides, cilindroides y la asociación de viarios de ellos, cada alumno elige uno para reproducirlo en maqueta. De este modo se suscita una reflexión constructiva y estructural, que aunque no son materias propiamente de primer curso, sirven para tematizar y avanzar problemáticas de la arquitectura y una formación técnica necesaria en el proceso de diseño arquitectónico.
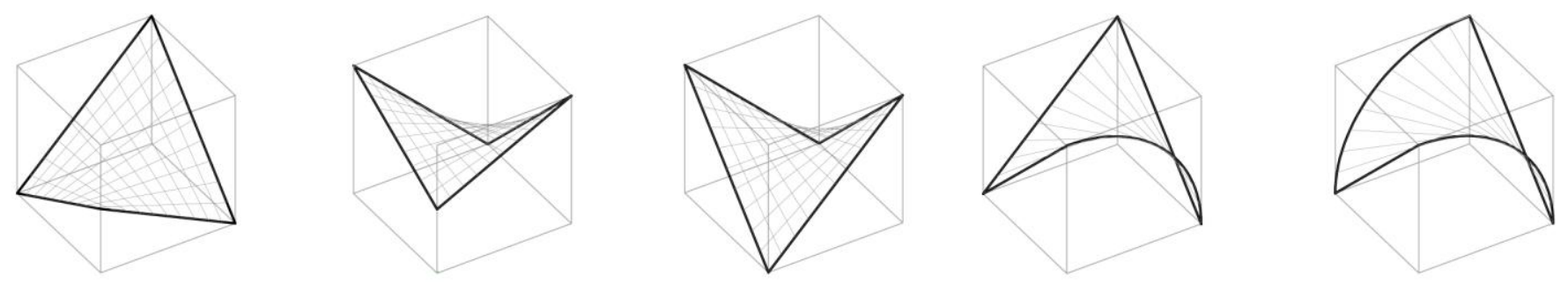

Fig 3. Representación de varias superficies regladas insertadas en un cubo. Paraboloides hiperbólicos, conoide y cilindroide. Dr.

Andrés Ros. Universidad Cardenal Herrera CEU.

El alumno debe establecer una estrategia constructiva y trazar un plan de diseño de la forma. Para ello en la asignatura se emplea la herramienta informática para dimensionar los diferentes elementos de la maqueta que posteriormente se cortarán con laser para generar las piezas. En este proceso de diseño de las barras, que forman parte del esqueleto portante de la superficie reglada, hay que prestar especial atención a la solución de los nudos en los que las barras se encuentran. Los diferentes ángulos que forman requieren soluciones especiales que aseguren la estabilidad del conjunto y la construcción real del modelo, presentando el problema como una casuística real a diferente escala.

Decidido el diseño se pasa a la siguiente fase de fabricación de las piezas mediante un proceso de CAD-CAM (Computer Aided Drawing y Computer Aided Manufacturing) es decir dibujo y fabricación asistida por ordenador. La secuencia de trabajo queda configurada así: 
Dibujo a mano bocetando las posibles soluciones de la superficie elegida. Dibujo por ordenador, atendiendo al dimensionado preciso de cada barra y la morfología y ángulos de las piezas especiales que actuarán de nudos de conexión y transmisión de cargas. Finalmente se procede al corte por laser y a la preparación del ensamblaje.
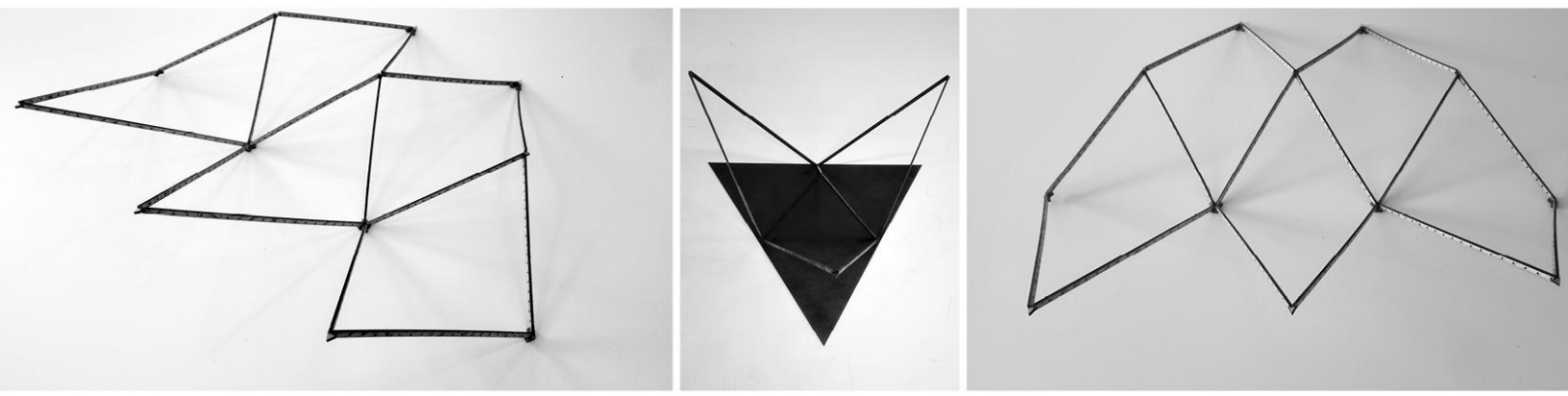

Fig 4. Esqueleto estructural construido por los alumnos para generar las superficies regladas.

Una vez obtenidas las piezas mediante corte por laser se ensamblarán generando el esqueleto estructural en cuya creación, el alumno ensaya la estabilidad y el proceso constructivo. Las barras deben haber sido calculadas con precisión para que todo encaje en el ensamblaje ya que, debido a la reducida escala de la maqueta, la tolerancia es mínima. Cada barra dispone de unas perforaciones cuyo número debe coincidir con el número de la barra opuesta y que servirán de guías para trazar las líneas generatrices de las superficies regladas. Estas líneas se resuelven con hilo que va cosiendo la estructura. Poco a poco el alumno va percibiendo la generación de la curva superficial fabricada mediante líneas rectas.

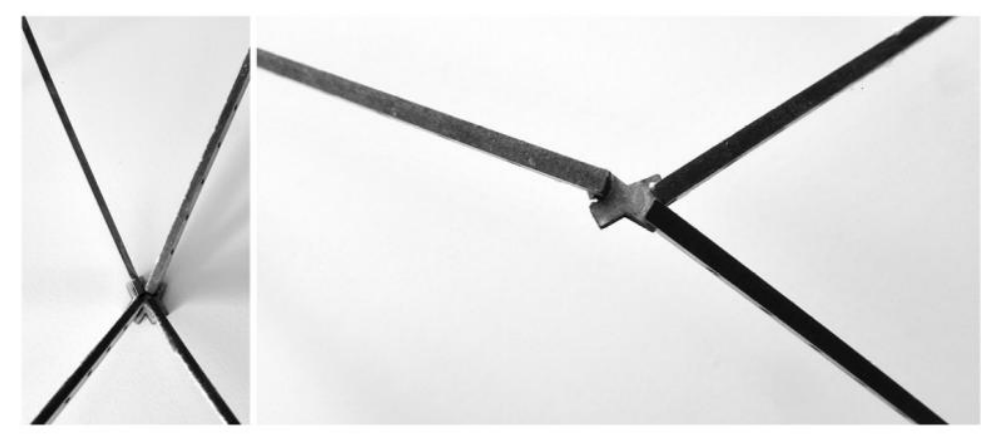

Fig 5. Nudo de encuentro de barras del esqueleto estructural. La complejidad de la unión obliga a diseñar una pieza especial que una las diferentes barras que se encuentran formando ángulos diferentes.

Hay que prestar especial cuidado en el encuentro de las barras que apoyan sobre la base, que requieren un apoyo sólido y estable. Como se aprecia en la imagen, esto se resuelve mediante una pieza que aloja unas perforaciones de espera para recibir cada una de las barras que apoyan sobre ella con un ángulo determinado. La complejidad del proceso propone un verdadero problema de diseño estructural, en el que se integran la física, la geometría, el dibujo y la capacidad técnica de dibujarlo, dimensionarlo y fabricarlo. 

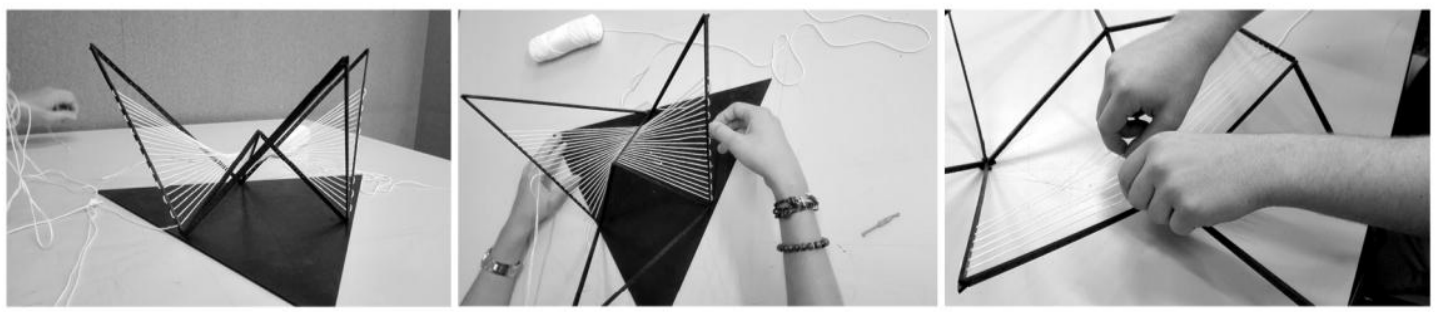

Fig 6. Proceso de generación de la superficie reglada sobre el esqueleto previamente construido.

Finalmente, una vez concluido el ejercicio el alumno es invitado a observar el resultado. Se propone una reflexión que sintetice el proceso seguido y para atestiguar las capacidades de visión y generación de la forma, adquiridas por el alumno, éste debe dibujar el resultado que, en comparación con el inicial difiere sustancialmente, ya que se constata que ahora el alumno está dotado de una capacidad de análisis y visión de la forma, que le ayuda a disponer de una estrategia de dibujo diferente. El alumno usa ahora en el proceso de dibujo la misma secuencia constructiva que ha trabajado para la realización de la maqueta. En primer lugar, realiza un encaje de la forma dentro del cubo que la contiene, dibujando el cubo "caja" en la perspectiva elegida y observada. Seguidamente sitúa en su interior las barras directrices de la superficie reglada y finalmente dividiendo las barras en el mismo número de segmentos, procede a unirlas mediante líneas rectas.

El resultado es un dibujo mucho más preciso, de una mejor ejecución gracias a la compresión de la generación de la forma. Hemos sido capaces de generar una construcción compleja partiendo del cuadrado elemental pasando por el cubo. Paso a paso hemos pasado del cuadrado al cubo como fundamento de la comprensión del proceso creativo. La lección aprendida defiende que en cualquier diseño arquitectónico hay un camino lógico que resuelve lo complejo. Una ecuación matemática compleja resuelta mediante geometría. Y lo que es más importante la existencia de una estrategia creativa necesaria en el proceso proyectual y como se ha demostrado varias disciplinas han participado de la práctica. Dibujar pensar analizar comprender resolver crear construir dibujar.
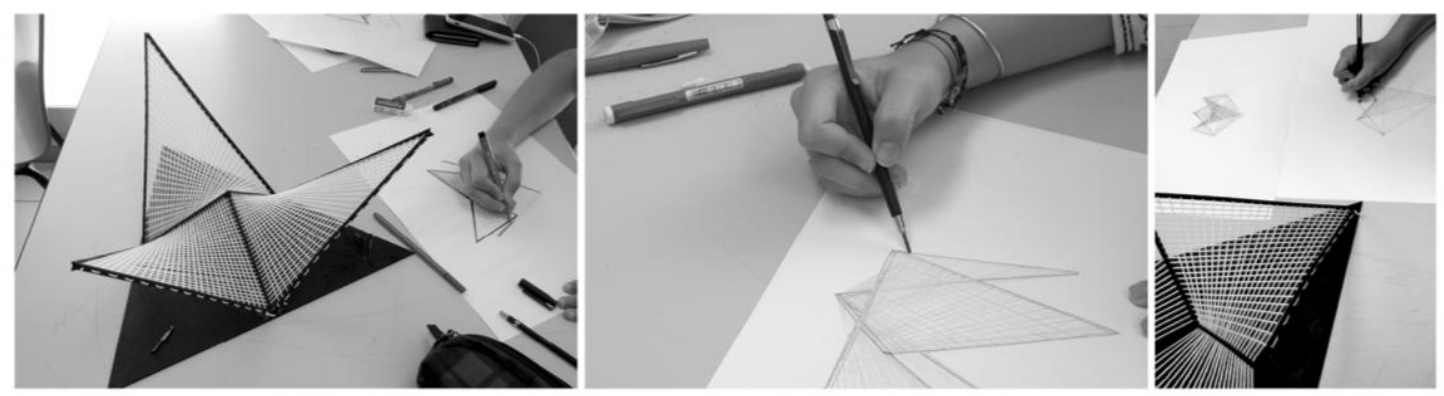

Fig 7. Dibujo final del resultado obtenido. Construir la maqueta de la superficie reglada ayuda a disponer de una estrategia de montaje que después sirve para disponer de una estrategia de dibujo. 


\section{Resultados, evaluación y conclusiones}

Durante el desarrollo de la experiencia piloto el alumno ha mostrado un interés por el ejercicio planteado asistiendo a clase con ganas de experimentar lo que la práctica proponía. Si el objetivo de la estrategia expuesta era romper las cajas aisladas que constituyen el programa académico del primer curso de arquitectura, los resultados esperados no pueden ser otros que los de mejorar la comprensión de la arquitectura como un hecho integral por parte del alumno. Los propios ejercicios planteados tienen implícito un enunciado en el que es obligado integrar conocimientos de otras áreas, por lo que superar dicho ejercicio significa demostrar que se ha obtenido el resultado deseado.
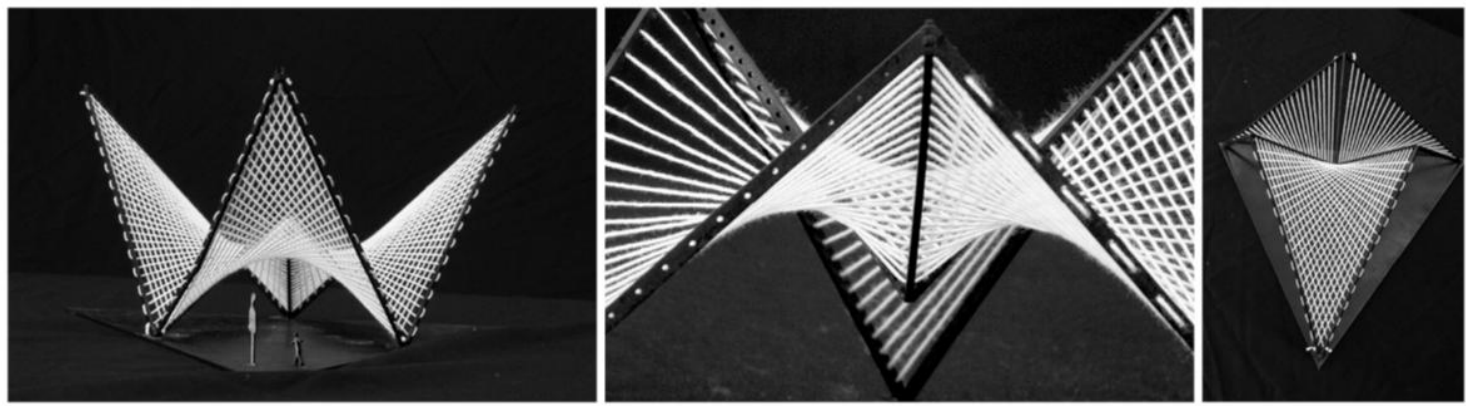

Fig 7. Maquetas construidas por los alumnos de primero de arquitectura.

No obstante, es importante reseñar que estos ejercicios no pretenden sustituir por completo aquellos intrínsecos de cada asignatura, sino complementarlos y conseguir una aplicación asociada al Grado al que sirven. Por consiguiente, serán ejercicios que convivan con otros que únicamente atañen a la materia en cuestión. La evaluación de cada asignatura queda de este modo sujeta a dos criterios complementarios también: el del profesor responsable de forma individual, que evaluará los ejercicios "propios" de dicha materia, y el del grupo de profesores con el que se comparte el ejercicio transversal.
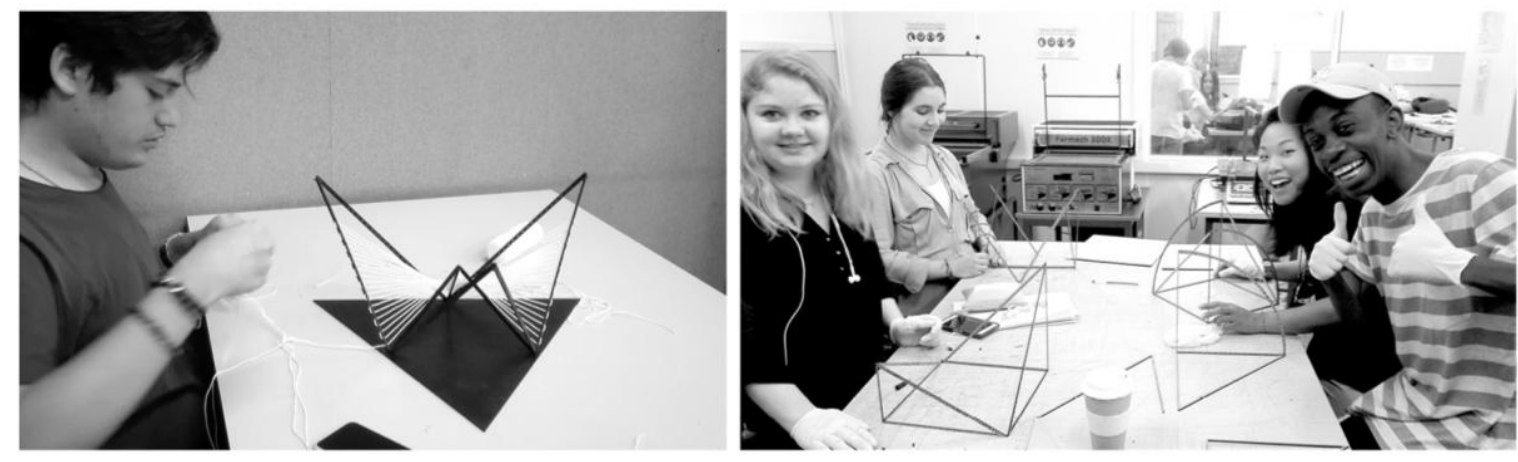

Fig 8. Proceso de construcción de las maquetas.

Asimismo, se propone una serie de indicadores que ayudarán a evaluar la bondad de la propuesta, tanto desde el punto de vista de la estrategia como de la aplicación concreta. Algunos de estos indicadores forman parte del sistema de garantía de calidad de la Escuela, y otros son de nueva incorporación. De la comparación de estos indicadores con los cursos 
anteriores a la implantación de la propuesta, y de los cursos sucesivos, se espera extraer conclusiones claras, objetivas y medibles que nos permitan a partir del curso 2019-20 profundizar en las acciones propuestas, o introducir acciones de mejora. Los indicadores son:

- $\%$ presentados en convocatoria ordinaria

- $\%$ presentados en convocatoria extraordinaria

- $\%$ aprobados en convocatoria ordinaria

- $\%$ aprobados en convocatoria extraordinaria

- $\%$ suspendidos en convocatoria ordinaria

- \% suspendidos en convocatoria extraordinaria

- \% asistencia regular a las clases

- $\%$ entrega en tiempo de trabajos transversales de las asignaturas

- $\quad \%$ entrega de trabajos transversales de las asignaturas

- nota media de los trabajos transversales

- nota media de los aprobados en convocatoria ordinaria

- nota media de los aprobados en convocatoria extraordinaria

- nota media de los suspensos en convocatoria ordinaria

- nota media de los suspensos en convocatoria extraordinaria

\section{Bibliografía}

AubreY, K. Y RILEY, A. Understanding \& Using Educational Theories. London: SAGE.

BLoom, B.S. Taxonomy of Educational Objectives: The Classification of Educational Goals. New York: David McKay Company, Inc.

BORDES, J. La infancia de las vanguardias: sus profesores desde Rousseau a la Bauhaus. 2007. Cátedra.

Franzen U., Pérez Gómez A., ShKapich K. 1999. Education of an architect: a point of view, the Cooper Union School of Art \& Architecture. Michigan. Monacelli Press.

Gropius, W. The New Architecture and the Bauhaus. 1965. MIT Press. 